\title{
Wavelength-controlled External-Cavity Laser with a Silicon Photonic Crystal Resonant Reflector
}

\author{
A. A. Gonzalez-Fernandez, Alexandros A. Liles*, Saydulla Persheyev, Kapil Debnath, \\ Liam O’Faolain \\ School of Physics and Astronomy, University of St Andrews, North Haugh, St Andrews KY16 9SS, \\ UK.; \\ *corresponding author: aal5@st-andrews.ac.uk
}

\begin{abstract}
We report the experimental demonstration of an alternative design of external-cavity hybrid lasers consisting of a III-V Semiconductor Optical Amplifier with fiber reflector and a Photonic Crystal (PhC) based resonant reflector on SOI. The Silicon reflector comprises a polymer (SU8) bus waveguide vertically coupled to a $\mathrm{PhC}$ cavity and provides a wavelength-selective optical feedback to the laser cavity. This device exhibits milliwatt-level output power and sidemode suppression ratio of more than $25 \mathrm{~dB}$
\end{abstract}

Keywords: silicon photonics, optical interconnect, photonic crystal, semiconductor laser

\section{INTRODUCTION}

Silicon photonics technology represents one of the most promising solutions to the fabrication of photonic integrated circuits (PIC). A particularly appealing feature of these is their support of Wavelength Division Multiplexing (WDM), which is a solution to the ever growing demands of bandwidth and integration in optical interconnections[1-4]. In order to exploit the advantages of WDM in Si photonics, it is crucial to obtain cheap, efficient, and Si-compatible tunable lasers with precise wavelength control [5].

While one of the most appealing technologies for Si PIC is the Silicon-On-Insulator platform, one of the elements not yet widely available in it is an efficient electrically pumped laser directly integrated in Si or other group IV elements. The use of III-V materials as gain elements the preferred solution to this issue. However, compatibility challenges and lattice mismatch makes this a difficult task. One approach to overcome such challenges is the use of direct wafer bonding for the heterogeneous integration of III-V elements on SOI-based systems [6]. An alternative solution is the formation of External-Cavity (EC) lasers by the use of external reflectors based on Si and Reflective Semiconductor Optical Amplifiers (RSOAs). The latter approach has the advantage of allowing independent design, fabrication and optimization cycles for the active and passive components, making a more effective use of the III-V materials [5, 7].

The present work demonstrates a novel EC laser design based on a low index waveguide coupled to a Photonic Crystal $(\mathrm{PhC})$ cavity which is used as a resonant reflector[8,9]. This represents a step forward in the pursuit of laser architectures with small footprint, high Side-Mode Suppression Ratios, and high precision peak wavelength control, all of which are requirements for efficient WDM applications [5].

\section{CONCEPT AND DESIGN}

The general design and working principle of the vertically coupled photonic crystal cavity and bus waveguide is detailed in [9]. A schematic of the system configuration presented in the figure 1a. Evanescent coupling of the light to the PhC cavity mode takes place at the resonance wavelength of the $\mathrm{PhC}$, which acts as a resonant mirror, providing the optical feedback by means of the light component that couples to the waveguide in the backward direction [8].

Optical Interconnects XVI, edited by Henning Schröder, Ray T. Chen, Proc. of SPIE Vol. 9753, 975317

(C) 2016 SPIE · CCC code: 0277-786X/16/\$18 · doi: 10.1117/12.2213288 
a)
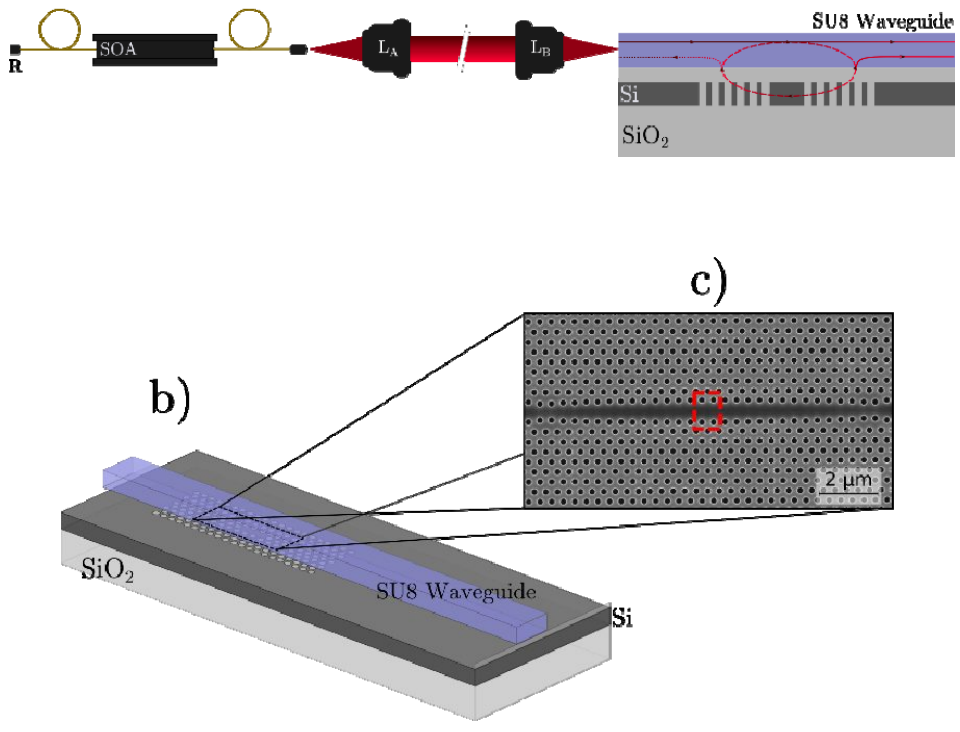

Figure 1. a) Schematic of the external laser cavity configuration coupled to the PhC DA cavity. b) Perspective schematic of the SOI chip containing the PhC DA cavity and waveguide. c) SEM image of one cavity. The holes inside the red square are those which position can be modified to control the resonance wavelength.

The nature of the vertical coupling allows for the maximization of the area available for the electronic components, as well as the isolation of these from the optical systems. This scheme also permits a better control of the coupling between the waveguide and Si by chip-scale optical links, while reducing the insertion and transmission losses.

When the SOA is electrically pumped, the broad IR light generated by spontaneous emission is coupled to the waveguide. Those wavelengths matching the resonance of the DA cavity will couple to it, and only these are going to be reflected due to the nature of the Si reflector. As represented in the figure 1a, this light will be guided back to the SOA thus forming a conventional laser cavity exclusively for such wavelengths. The laser output is collected at the other end of the waveguide.

Under the weak coupling approximation, the effective transmittance $(\mathrm{T})$ and reflectance $(\mathrm{R})$ in the waveguide can be derived from the intrinsic $\mathrm{Q}$-factor of the $\mathrm{PhC}$ cavity $\left(\mathrm{Q}_{\text {cavity }}\right)$ and the $\mathrm{Q}$-factor related to its coupling to the waveguide $\left(\mathrm{Q}_{\text {coupling }}\right)$ as described in [11]. Then, the value of $\mathrm{R}$ for can be tailored as needed by the modification of the PhC cavity design, thus modifying the laser output wavelength. The relatively large length of the laser cavity (between $5 \mathrm{~m}$ and $8 \mathrm{~m}$ ) results in an effective longitudinal mode continuum, as this causes a significantly narrow longitudinal mode spacing (sub femtometre).

The output wavelength is determined by the $\mathrm{PhC}$ cavity design, resulting in the laser cavity length variations having minimal impact on it, delivering a flexible system in this regard. In addition, the high control over the PhC constant allowed by its fabrication method results in the most precise control of the emitted wavelength achieved to date in hybrid $\mathrm{Si}-\mathrm{III} / \mathrm{V}$ external cavity lasers (in the order of $\mathrm{nm}$ ).

\section{EXPERIMENTAL}

The gain medium used for the configuration represented in the figure 1 was a Kamelian OPA-20-N-C-FA fiber-pigtailed SOA (minimum fiber-to-fiber gain of $20 \mathrm{~dB}$ for $\lambda \sim 1550 \mathrm{~nm}$ ). As the figure 1a depicts, the light from the SOA was coupled to the waveguide using a free-space configuration consisting of two lenses $\mathrm{L}_{\mathrm{A}}$ and $\mathrm{L}_{\mathrm{B}}$ to respectively collimate and focus the light on the waveguide to couple it to the resonant reflector device that will function as the one of the mirrors of the laser cavity. The second mirror was a commercial fibre optic reflector $(\mathrm{R})$ with reflectance $\mathrm{R} \sim 95 \%$. The $2.1 \mu \mathrm{m}$ height and $3 \mu \mathrm{m}$ wide bus waveguide was fabricated using SU8 Polymer and coupled to a Dispersion Adapted 
(DA) PhC cavity[10] as described in [9] (figures $1 \mathrm{~b}$ and 1c). The PhC was fabricated using a $220 \mathrm{~nm}$-thick SOI wafer. The structure was defined using Electron-beam Lithography to define de pattern in ZEP, and etching the Si by Reactive Ion Etching. Spin-on-glass technique with Flowable Oxide by Dow Corning (Fox-15) was used to fabricate the buffer oxide layer. Finally, the facets of the device were coated with an $\mathrm{MgF}_{2}$ anti-reflective film. Details on the DA cavity fabrication can be found in [10].

\section{RESULTS AND DISCUSSION}

External cavity lasers with the configuration depicted in the figure 1a were tested using different PhC cavity designs. A double lens configuration equivalent to that described in the same figure was focused to the other end of the waveguide to characterize the output power values and spectra. These were respectively obtained connecting the fibre to a THORLABS Power Meter PM100D using a S122C sensor, and an Advantest Optical Spectrum Analyzer with a maximum resolution of $0.1 \mathrm{pm}$. Different SOA driving currents where used to obtain different sets of data. In order to verify the resonance wavelengths of the cavities as passive devices, non-lasing tests were also performed substituting the SOA in the input by an Amonics ALS-CL-15-B-FA IR Broadband Source.

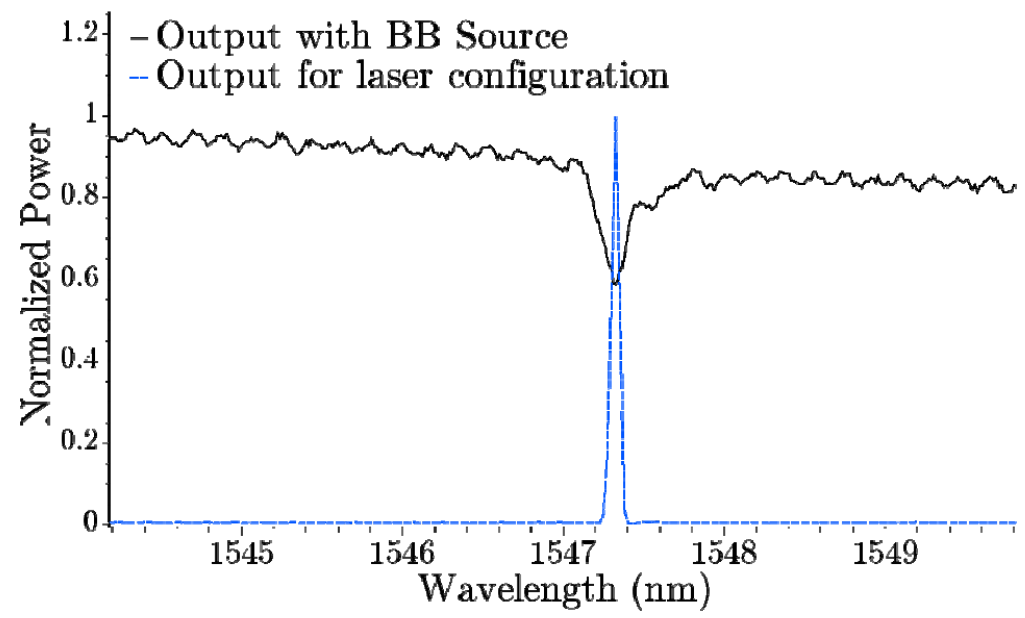

Figure 2. Normalized output spectra for the configuration described in the figure 1a (dashed line) and for the passive characterization using a broadband (BB) source in the input of the system (continuous line).

The figure 2 presents the normalized power detected by the spectrometer versus wavelength for a device under test consisting of a PhC cavity with a period lattice constant $\alpha=388 \mathrm{~nm}$ and radius to period ratio of $\mathrm{r} / \alpha=0.28 \mathrm{~nm}[10]$. The graph shows the results for both the passive resonance characterization using the broadband source (black continuous line), and for the output of the cavity laser configuration (dashed blue line). The clear match of the laser configuration wavelength to the resonances of the $\mathrm{PhC}$ cavity can be corroborated in this figure.

The figure 3 shows the Power vs Current results for the laser in this configuration. The threshold current was $23.6 \mathrm{~mA}$ when lasing at a wavelength of $1547.32 \mathrm{~nm}$, and the efficiency is $\eta_{\mathrm{s}}=0.032 \mathrm{~mW} / \mathrm{mA}$. The mirror reflectivity and losses of the $\mathrm{PhC}$ cavity are respectively $\sim 40 \%$ and $\sim 7.5 \mathrm{~dB}$, as calculated using the experimental threshold gain and slope efficiency [12]. 


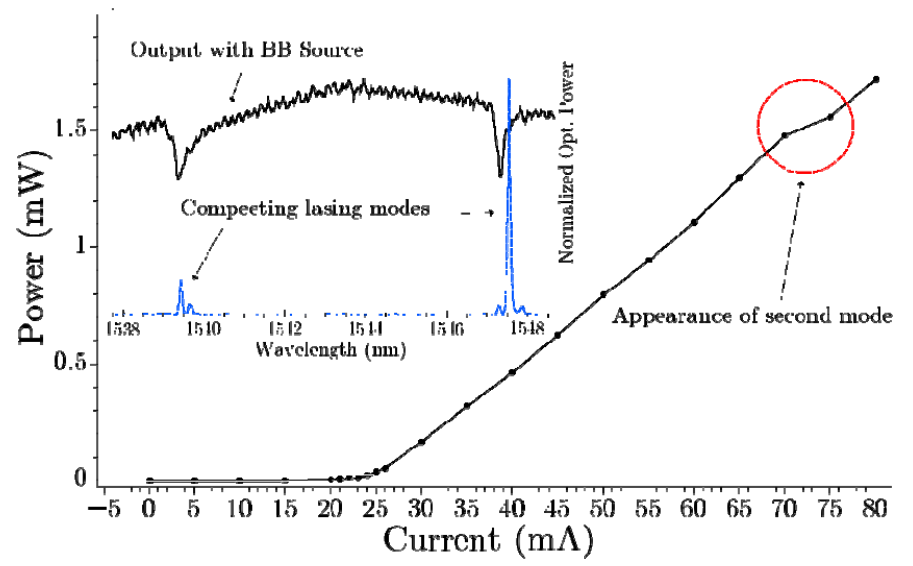

Figure 3. Power Vs Current (P-I) of the Cavity Laser. The inset shows the PhC cavity resonances (black continuous line) along with the competition of two lasing modes (blue dashed line). The highlighted kink in the P-I curve shows the point in which dual lasing appears, causing disruptions on the trend of the curve for single mode lasing.

The feature highlighted with a red circle in the figure 3 is the point in which the lasing shifts to a second mode corresponding to other resonance wavelength of the $\mathrm{PhC}$ cavity. The inset in the same figure shows the two competing lasing modes. Note once again that each mode matches one $\mathrm{PhC}$ cavity resonance. Such coexistence or shifting of modes is due to the effective creation of more than one laser cavity resulting from the multimodal character of the DA cavities. Each coupling mode acts as an individual wavelength-selective reflector with particular values for $\mathrm{R}$ and $\mathrm{T}$ according to the wavelength and Q-factor of the corresponding PhC cavity mode. Then, the laser cavities are de-coupled from each other, meaning that each one has its own threshold voltage, efficiency, etc.

In the case of the device discussed so far, the current threshold for lasing for the second mode was $68 \mathrm{~mA}$, where the kink appears in the figure 3 . As multiple lasing modes may be an undesired feature in completed devices, a straightforward approach to avoid them would be to simply use a single-mode PhC cavity design.

In order to explore the wavelength control that can be achieved in the presented system by lithographic means, different $\mathrm{PhC}$ cavities were fabricated and tested in the configuration. In order to modify the resonance frequencies, the only change done was the variation of the separation of the four inner holes of the DA cavity highlighted in the figure 1c[10]. The figure 4 shows the results from four designs, starting from a distance between the holes defined as $\mathrm{i}$, and progressively increasing it to $i+8, i+12$, and $i+16$. A clear change in the lasing wavelength can be observed, with sidemode suppression values above $25 \mathrm{~dB}$ in all the cases.

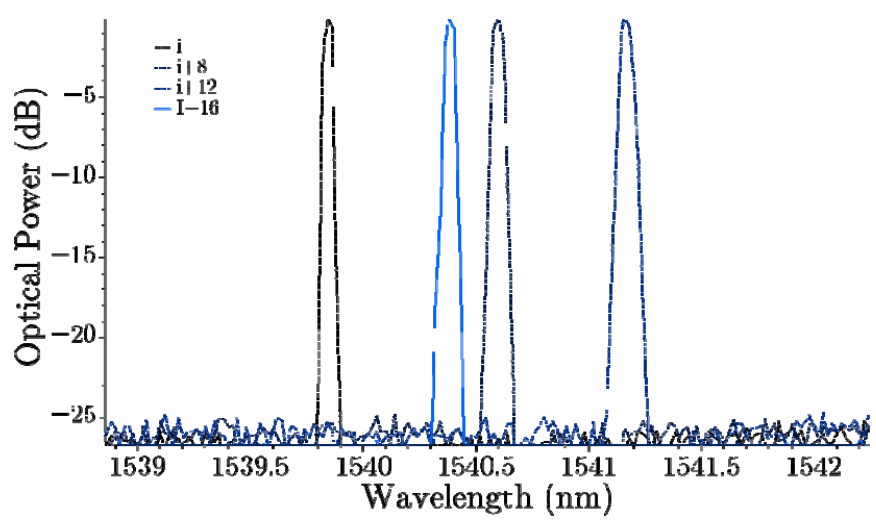

Figure 4. Spectra of laser cavities with different PhC DA cavity designs. The output wavelength changes due to the different resonances presented by each design. 
In order to reduce the overall footprint of the system and take a step forward to the integration of the laser cavity, a configuration with a butt-coupled IR Reflective SOA (RSOA) provided by CST Global was also tested. The optical amplifier was electrically driven using a Keithley 2460 source meter. The figure 5 shows a schematic of this configuration.

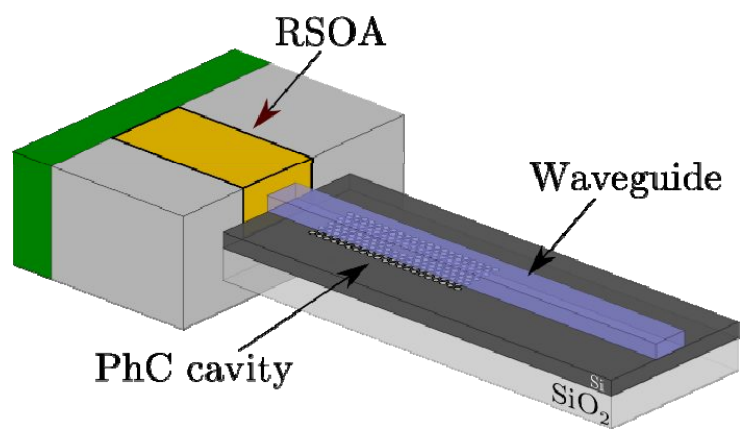

Figure 5. Schematic of the but-coupled configuration of the laser cavity.

The principle is the same as the previously analyzed, but one of the challenges is to properly couple the RSOA output to the SU8 waveguide. This was achieved using a micro-positioning system to place the RSOA at the point that delivered the highest power value in the output of the waveguide below lasing threshold. The RSOA was AR-coated with a film optimized for the semiconductor-air interface, in order to avoid parasitic reflections that would disturb the control of the lasing wavelength.

The figure 6 presents the laser spectra for the but-coupled configuration and its power-current characteristics. The lasing threshold was between $10 \mathrm{~mA}$ and $15 \mathrm{~mA}$, getting output power up to $1.2 \mathrm{~mW}$. The side mode suppression values were comparable or better than in the pig-tailed configuration, with values of $\sim 35 \mathrm{~dB}$, and lasing on a single longitudinal mode was achieved. Note that there is a more pronounced ripple in the base line of the spectrum as compared to the larger footprint system. This is caused by deficiencies in the AR coating of the RSOA, indicating there still is room for improvement in that regard.
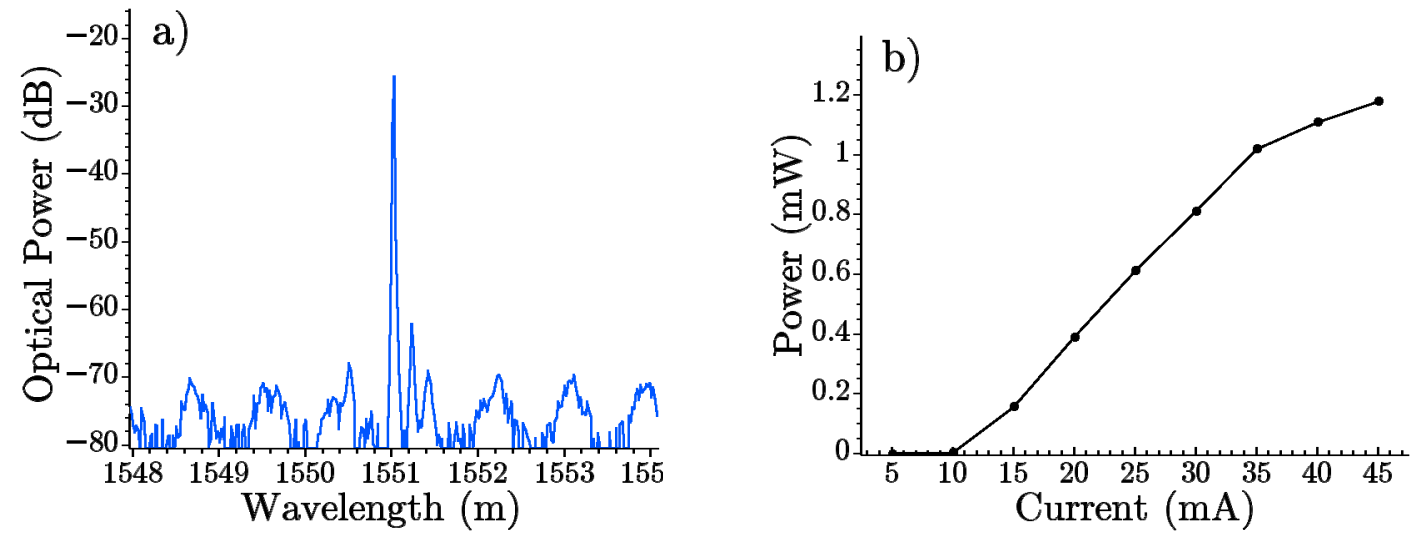

Figure 6. Spectrum (a) and Power vs Current (b) behavior of the laser cavity in the but-coupled configuration schematized in the figure 5 .

\section{CONCLUSIONS}

A hybrid external-cavity laser based on the use of a $\mathrm{PhC}$ cavity vertically coupled to a polymer guide as $\mathrm{Si}$ reflector is demonstrated for the first time. The configuration uses a fibre pigtailed SOA with a fibre optical reflector, detaching the wavelength selective components and the gain section. This separates the power consumption of the latter from the onchip elements, reducing thermal stability issues, while keeping as on-chip all the elements related to the wavelength 
tuning and control. Intra cavity losses (coupling and waveguide losses) of $\sim 7.5 \mathrm{~dB}$ were observed, and a reduction to as low as $3 \mathrm{~dB}$ is anticipated if the free space characterization set-up is optimized. Likewise, an improvement of the efficiency by a factor of $\sim 5$ is expected.

The same concept was used to obtain a small footprint cavity laser using a commercial RSOA butt-coupled to the PhC device. Lasing matching the DA cavity wavelength was also obtained, achieving values for power and sidemode suppression of $\sim 1.2 \mathrm{~mW}$ and $\sim 35 \mathrm{~dB}$, respectively.

Since $\mathrm{PhC}$ cavities show extremely small footprint and wide free spectral range as a result of their high $\mathrm{Q} / \mathrm{V}$ ratio[13], the architecture here presented an ideal candidate for WDM applications, especially in conjunction with other PhC cavity solutions $[14,15]$. However, the flexibility of the configuration also make them suitable for several different applications, including other light-based communications and sensing [16].

\section{Acknowledgements}

This work was partially funded by the EPSRC, Scottish Enterprise, and the European Research Council under the Starting Grant 337508.

\section{REFERENCES}

[1] Y. Vlasov, "Silicon CMOS-integrated nano-photonics for computer and data communications beyond 100G," Communications Magazine IEEE 50, s67-s72 (2012)

[2] A. V. Krishnamoorthy, R. Ho, X. Zheng, H. Schwetman, J. Lexau, P. Koka, GuoLiang Li, I. Shubin, and J. E. Cunningham, "Computer systems based on silicon photonic interconnects," Proc. IEEE 97(7), 1337-1361 (2009)

[3] B. Jalali and S. Fathpour, "Silicon photonics," J. Lightwave Technol. 24(12), 4600-4615 (2006).

[4] R. A. Soref, "The past, present and future of silicon photonics", IEEE J. Sel. Top. Quant., 12(6), 1678-1678 (2006)

[5] X. Zheng, S. Lin, Y. Luo, J. Yao, G. Li, S. S. Djordjevic, J. H. Lee, H. D. Thacker, I. Shubin, K. Raj, J. E. Cunningham, and A. V. Krishnamoorthy, "Efficient WDM laser sources towards terabyte/s silicon photonic interconnects," J. Lightwave Technol. 31(24), 4142-4154 (2013)

[6] G. Roelkens, L. Liu, D. Liang, R. Jones, A. Fang, B. Koch, and J. Bowers, Laser Photon. Rev. 4, 751 (2010).

[7] A. J. Zilkie, P. Seddighian, B. J. Bijlani, W. Qian, D. C. Lee, S. Fatholoumi, J. Fong, R. Shafiiha, D. Feng, B. J. Luff, X. Zheng, J. E. Cunningham, A. V. Krishnamoorthy, and M. Ashgari“Power-efficient III-V/Silicon external cavity DBR lasers“, Op. Express 20, Issue 21, pp 23456-23462 (2012).

[8] M. Qiu, "Vertically coupled photonic crystal optical filters", Op. Lett. 30, Issue 12, pp 1476-1478 (2005)

[9] K. Debnath, K. Welna, M. Ferrera, K. Deasy, D. G. Lidzey, and L. O'Faolain, "Highly efficient optical filter based on vertically coupled photonic crystal cavity and bus waveguide", Op. Lett. 38, Issue 2, pp 154-156 (2013)

[10] K. Welna, S. L. Portalupi, M. Galli, L. O'Faolain, and T. F. Krauss, "Novel dispersion-adapted photonic crystal cavity with improved disorder stability", IEEE J. Quant. Electron. 48, No 9, pp 1177-1183 (2012)

[11] Y. Xu, Y. Li, R. K. Lee, and A. Yariv, "Scattering-theory analysis of waveguide-resonator coupling", Phys. Rev. E 62, No 5, pp 7389-7404 (2000)

[12]L. A. Coldren, S. W. Corzine, M. L. Mashanovitch, "Diode lasers and photonic integrated circuits", 2nd Edition, Wiley, New York (2012)

[13] M. Notomi, K. Nozaki, A. Shinya, S. Matsuo, and E. Kuramochi, "Toward fJ/bit optical communication in a chip", Opt. Commun. 314, pp 3-17 (2014)

[14] K. Debnath, F. Y. Gardes, A. P. Knights, G. T. Reed, T. F. Krauss \& L. O’Faolain "Dielectric waveguide vertically coupled to all-silicon photodiodes operating at telecommunication wavelengths," Applied Physics Letters 102, 171106 (2013)

[15] K. Debnath, L. O'Faolain, F. Y. Gardes, A. G. Steffan, G. T. Reed \& T. F. Krauss "Cascaded modulator architecture for WDM applications," Optics Express 20, 27420-27428 (2012)

[16] W. Zeller, L. Naehle, P. Fuchs, F. Gerschuetz, L. Hildebrandt \& J. Koeth "DFB Lasers Between 760 nm and 16 $\mu \mathrm{m}$ for Sensing Applications," Sensors 10, 2492-2510 (2010). 